\title{
Resistance to extinction in classical GSR conditioning as a function of acquisition trials beyond peak CR size'
}

\author{
A. I. SIL VER, Ohio University, Athens, Ohio 45701 and H. D. \\ KIMMEL, University of South Florida, Tampa, Fla. 33620
}

Seventy-two Ss were randomly divided into six groups, five for classical delayed GSR conditioning and one an unpaired control. The conditioning groups received either $1,2,4,8$, or 16 paired trials after the peak $C R$ and the controls were matched to Group 16 in number of CSs and UCSs received unpaired. The CS was a 1000-cps pure tone and the UCS a shock to the forearm, with a 5-sec CS-UCS interval. An inverse relationship between resistance to extinction and number of postpeak conditioning trials was found for both trials and magnitude data, except for Group 1. It was concluded that inhibition of delay combines with extinctive inhibition to hasten extinction and that the two types of inhibition may be identical.

A novel procedure was used in this study to define the number of classical conditioning acquisition trials given to each $S$, calculated to result in better control of the degree of inhibition of delay generated than is obtained in studies using fixed numbers of trials. When the $S$ first made a $C R$ which was smaller than his immediately preceding CR, the preceding CR was designated as the "peak CR." The independent variable manipulated was the number of paired-acquisition trials given following this peak CR. Assuming that the peak CR would reliably identify a common point (among Ss) in the development of inhibition of delay, and assuming that inhibition of delay would facilitate extinction, it was hypothesized that resistance to extinction would vary inversely with the number of postpeak-acquisition trials.

\section{SUBJECTS AND INSTRUCTIONS}

Seventy-two undergraduate and high school students served as $\mathrm{Ss}$, the youngest being 16 years old. The Ss were assigned at random to six groups, separately for each sex. Three Ss were dropped because of $\mathrm{E}$ errors (no two from the same group). The groups differed according to whether they received one paired trial following the peak CR (Group 1), two paired trials CR (Group 2), four (Group 4), eight (Group 8), 16 (Group 16), or unpaired CSs and UCSs which were matched in number with those given to the members of Group 16 (Group C).

The Ss were told that this was a study of the effects of external stimuli on the GSR and that they were to remain alert while avoiding unnecessary movements.

\section{APPARATUS}

Data were collected in a darkened IAC chamber containing an armchair and an intercom. The CS was a 40-dB (physical reference), 1000-cps tone, of $5.0 \mathrm{sec}$ duration. The UCS, a dc shock via $3 / 4$-in. zinc electrodes to the volar surface of the left forearm, had an intensity of $4.0 \mathrm{~mA}$ and a duration of $0.1 \mathrm{sec}$. The CS-UCS interval was $5.0 \mathrm{sec}$ and the intertrial interval was varied between $30-70 \sec (\overline{\mathrm{X}}=50)$.

The GSR was picked up as a dc resistance change from the palm and back of the right hand by $3 / 4$-in. zinc-zinc sulphate electrodes in Lucite cups filled with saline GSR paste. It was amplified by a Biophysical Instruments Company GSR amplifier and recorded on a Texas Instruments Company Recti-Riter with a paper speed of $3 \mathrm{in} . / \mathrm{min}$.

$$
\text { PROCEDURE }
$$

The electrodes were attached, the $S$ was seated in the chamber, the lights turned off, and the door closed. Instructions were read over the intercom. Five minutes later the $\mathrm{S}$ was given three trials of UCS alone and as many CSs as were necessary to result in two consecutive zero responses (maximum of 10). The conditioning trials followed immediately, each $\mathrm{S}$ receiving as many postpeak pairings as was appropriate in his group. Ss in Group C were matched to those in Group 16 to receive the same number of CSs and UCSs in an unpaired sequence. All Ss then received as many CS-only trials as were needed to achieve an extinction criterion of two consecutive zero responses (maximum of 50 ).

\section{RESULTS}

The GSR was transformed to units of change in log conductance for analysis. A comparison was made between the combined paired groups and Group $\mathrm{C}$ during acquisition to determine that conditioning had, in fact, occurred. Since the groups received different numbers of paired trials, this comparison used only the peak CR and the CR on the next trial. The difference favoring the conditioning groups over Group $C$ was highly significant $[t(67)=2.76, p<.01]$.

Resistance to extinction was measured in terms of the number of trials to criterion, as shown in Fig. 1. A horizontal line representing Group $\mathrm{C}$ is drawn in the figure for comparison with each of the conditioning groups.

Except for Group 1, the data in Fig. 1 were in accord with the hypothesis of an inverse relationship between resistance to extinction and number of postpeak acquisition trials. The differences among the groups' means were highly significant, $F(5,63)=4.05$, MS(error) $=274.4$. Duncan's test revealed that Group 2 was significantly higher than Groups 8,16 , and $\mathrm{C}$, but that none of the other groups differed significantly.

Figure 2 shows the average GSR magnitude in extinction for each of the conditioning groups, in five blocks of two trials (after this the Ns were too small). Again, except for Group 1, the data were in accord with the hypothesis. Groups 2 and 4 made substantially larger GSRs during extinction than did the other groups and, in addition, unusual persistence in responding was shown. The conditioning groups differed very significantly in extinction, $\mathrm{F}(4,52)=18.00$, MS(error) $=.001$. None of the other effects were significant. Duncan's test showed that Group 2 was significantly higher than Groups 1, 8 , and 16 , Group 4 was significantly higher than Group 16. and none of the other groups differed significantly.

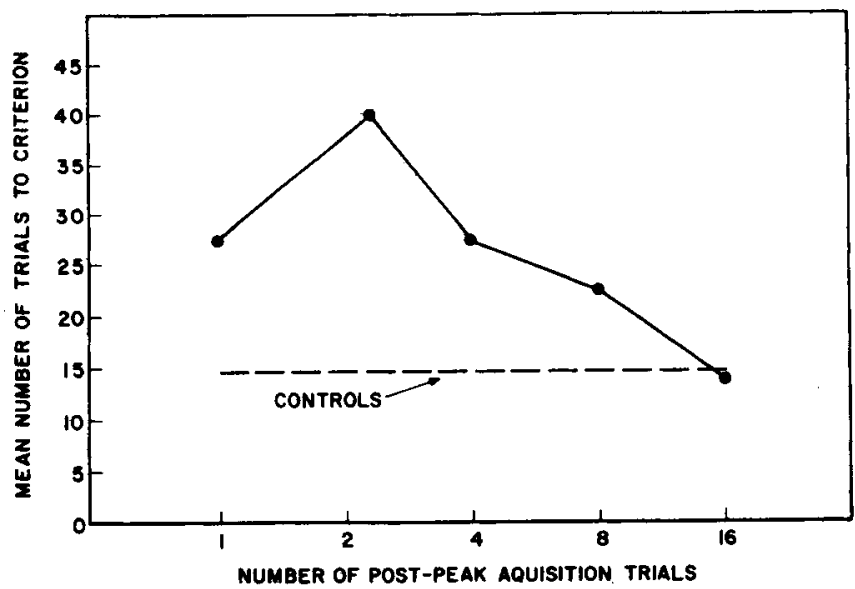

Fig. 1. Mean number of trials to extinction criterion for the five conditioning groups and for the controls (shown as a horizontal line in the figure).

(Continued on page 55) 